\title{
Mucopolysaccharides in Urine during Normal Human Development
}

\author{
Carolyn Douglas, Judith Nowak, and B. Shannon Danes ${ }^{[16]}$ \\ Division of Human Genetics, Department of Medicine, Cornell University Medical College, New York, New York, USA
}

\begin{abstract}
Extract
To determine the pattern of urinary mucopolysaccharides during normal human development, the urine samples from 92 normal individuals (aged from birth to 25 years) were analyzed for degradation ratio (large molecular weight mucopolysaccharides/fragments) and for the electrophoretic pattern of the different mucopolysaccharides present. The degradation ratio averaged $0.42 \pm 0.21$ (range 0.13-0.75) and was independent of age and sex. It was determined by electrophoresis that the major mucopolysaccharide was chondroitin sulfate. In the eight patients with a genetic mucopolysaccharidosis the degradation ratio was above 1.0 and electrophoresis revealed the presence of large quantities of dermatan sulfate and heparan sulfate. The two tests, therefore, permit distinction of urine from a normal individual from that of a patient with a genetic mucopolysaccharidosis.
\end{abstract}

\section{Speculation}

On the basis of studies done on cultured fibroblasts, it is apparent that the term genetic mucopolysaccharidosis encompasses a much broader base of cellular pathology than that previously considered. Normal values for urinary mucopolysaccharides throughout development in conjunction with cell culture studies are needed by clinicians trying to determine whether a patient with some but not all the stigmata suggestive of a genetic mucopolysaccharidosis has a disorder of mucopolysaccharide metabolism.

\section{Introduction}

Qualitative and quantitative analyses of urinary mucopolysaccharide excretion have provided variables which reflect the mucopolysaccharide metabolism of the body $[4,6]$.

Previous studies $[4,10]$ have attempted to establish values for total urinary mucopolysaccharide excretion in terms of total uronic acid per $24 \mathrm{hr}$ or per milligram of creatinine. Difficulty has been experienced, however, in establishing meaningful values. Thus, Di Ferrante et al. [3] proposed that the degradation ratio defined as the large molecular weight mucopolysaccharides/fragments was a more sensitive reflection of mucopolysaccharide metabolism. They reported that the ratio was less than 1 in normal individuals and consistently greater than 1 in children affected with various mucopolysaccharide-storage disorders. It was therefore concluded that the degradation ratio was a reliable index of mucopolysaccharide metabolism and that any increase in the ratio above the normal range indicated a disorder in degradation of mucopolysaccharides. Unfortunately, standard values for a group of normal individuals have not been available. 
The purpose of the present study was to establish the pattern of urinary mucopolysaccharide excretion for a group of normal individuals (aged from birth to 25 years) with two tests: (1) the degradation ratio to establish standard values for the normal population and (2) electrophoretic profiles of purified urinary mucopolysaccharides isolated from the same normal individuals.

\section{Materials and Methods}

Urine samples from 92 normal individuals of both sexes whose ages ranged from several weeks to 25 years and from 8 patients with different genetic mucopolysaccharidoses were collected without preservative, measured, and filtered. Urinary mucopolysaccharides were precipitated with $1 \%$ cetylpyridinium chloride (CPC). The urinary mucopolysaccharide fragments not precipitated by the detergent were passed through a Dowex-50 column, collected on a column of Dowex-1 $2 \mathrm{X}$ resin (200-400 mesh, $\mathrm{Cl}$ form) and eluted in various fractions with step-wise increasing concentrations of $\mathrm{NaCl}$ [2]. Both the large molecular weight urinary mucopolysaccharides in the precipitate and the fragments in the supernatant were estimated as uronic acid by the carbazole method [5]. Reproducibility of recovery was measured by duplicate isolations of mucopolysaccharides from urine samples from four normal individuals.

The electrophoretic pattern of the purified CPC-precipitated mucopolysaccharides was observed on cellulose acetate strips in a Beckman microzone cell [12] using $0.1 \mathrm{~m}$ barium acetate buffer to identify individual mucopolysaccharides [11]. In this buffer system heparan sulfate and chondroitin sulfate migrated separately; hyaluronic acid migrated with dermatan sulfate.

\section{Resulls and Discussion}

Urinary mucopolysaccharides have been characterized quantitatively by 24-hr total excretion [4, 10] and qualitatively by the distribution of different mucopolysaccharides $[4,6,10,11]$. An alteration in either variable has been considered to be suggestive of a mucopolysaccharide disorder. To interpret an alteration in ratios it is, of course, necessary to know the range of ratios for normal individuals of the same sex and age. Recent investigations [1] have shown that at least some of the genetic mucopolysaccharidoses are due to deficiencies in specific degradative enzymes and measurement of
Table I. Mucopolysaccharide degradation [2, 3] (large molecular weight mucopolysaccharides/fragments) of urine samples from normal subjects of both sexes ${ }^{1}$

\begin{tabular}{|c|c|c|c|c|c|}
\hline \multirow{2}{*}{ Age, yr } & \multirow{2}{*}{ Sex } & \multirow{2}{*}{$\begin{array}{l}\text { No. of } \\
\text { Persons }\end{array}$} & \multicolumn{3}{|c|}{ Mucopolysaccbride Degradation ratios } \\
\hline & & & Mean & SD & Range \\
\hline \multirow[t]{2}{*}{$0-1$} & M & 6 & 0.61 & 0.27 & $0.15-0.99$ \\
\hline & $\mathrm{F}$ & 3 & 0.55 & 0.30 & $0.48-0.69$ \\
\hline 2 & M & 4 & 0.43 & 0.19 & $0.20-0.62$ \\
\hline \multirow[t]{2}{*}{3} & M & 3 & 0.44 & 0.07 & $0.32-0.72$ \\
\hline & $\mathrm{F}$ & 2 & 0.40 & 0.20 & $0.34-0.68$ \\
\hline 4 & $\mathbf{M}$ & 4 & 0.44 & 0.30 & $0.27-0.61$ \\
\hline \multirow[t]{2}{*}{$5-10$} & M & 12 & 0.39 & 0.25 & $0.19-0.75$ \\
\hline & $\mathrm{F}$ & 11 & 0.37 & 0.18 & $0.13-0.72$ \\
\hline \multirow[t]{2}{*}{$10-15$} & M & 13 & 0.44 & 0.12 & $0.18-0.72$ \\
\hline & $\mathrm{F}$ & 9 & 0.40 & 0.15 & $0.25-0.74$ \\
\hline \multirow[t]{2}{*}{$15-20$} & $M$ & 6 & 0.46 & 0.14 & $0.14-0.63$ \\
\hline & $\mathrm{F}$ & 10 & 0.36 & 0.26 & $0.16-0.64$ \\
\hline \multirow[t]{2}{*}{$20-25$} & $\mathrm{M}$ & 4 & 0.33 & 0.21 & $0.17-0.48$ \\
\hline & $\mathrm{F}$ & 5 & 0.38 & 0.24 & $0.15-0.54$ \\
\hline
\end{tabular}

${ }^{\mathrm{I}} \mathrm{M}$ : male; $\mathrm{F}$ : female.

these enzymatic activities is of more precise diagnostic value.

Duplicate isolation of mucopolysaccharides, both large molecular weight and fragments, from different urine samples from four normal individuals showed that recovery had an experimental error of less than $5 \%$. Table I lists the degradation ratios with range for age (by years and sex). During the 1st year of life the urinary degradation ratios of nine normal individuals were below 1.00. For the urine samples from 83 normal individuals between 2 and 25 years of age, the degradation ratio averaged $0.42 \pm 0.21$ (range $0.13-0.75)$. This ratio was not influenced by age, sex, or puberty (Table II).

The urinary mucopolysaccharide ratios of the eight

Table II. Statistical analyses: A comparison of the degradation ratio of urine samples from pre- and postpubescents and in males and females

\begin{tabular}{llllll}
\hline \multirow{2}{*}{ Subject } & $\begin{array}{c}\text { No. of } \\
\text { determin- } \\
\text { ations }\end{array}$ & \multicolumn{4}{c}{ Degradation ratios $[2,3]$} \\
\cline { 5 - 6 } & & Mean & $t$ & $P$ & $2 P$ \\
\hline $\begin{array}{c}\text { Ratio of pre-/post- } \\
\text { pubescent }\end{array}$ & $82 / 37$ & $0.44 / 0.38$ & 0.5044 & $0.70>2 P^{\prime}>0.60$ \\
$\begin{array}{c}\text { Ratio of males/ } \\
\text { females }\end{array}$ & $65 / 54$ & $0.40 / 0.41$ & 0.1043 & $0.90>2 P>0.80$ \\
\hline
\end{tabular}


Table III. Urinary mucopolysaccharide degradation ratio (large molecular weight mucopolysaccharides/fragments) of urine samples from patients with different mucopolysaccharide disorders

\begin{tabular}{|c|c|c|c|}
\hline Mucopolysaccharidoses & Subject & Age, yr & $\begin{array}{c}\text { Ratio } \\
\text { (large } \\
\text { molecular } \\
\text { weight/ } \\
\text { fragments) }\end{array}$ \\
\hline \multirow[t]{3}{*}{ Hurler } & $B B$ & 6 & 3.34 \\
\hline & $C S$ & 3 & 2.25 \\
\hline & $P S$ & 0.5 & 2.43 \\
\hline Hunter & $J B$ & 2 & 1.89 \\
\hline \multirow[t]{3}{*}{ Sanfilippo } & $C C$ & 3 & 2.25 \\
\hline & $D C$ & 5 & 2.43 \\
\hline & $D F$ & 9 & 3.22 \\
\hline Compound [9] Hurler/Scheie & $J A$ & 3 & 1.16 \\
\hline
\end{tabular}

patients with a genetic mucopolysaccharidosis were all above 1 (Table III). There was a good correlation between clinical stigmata reflecting tissue storage and increase in degradation ratio. The patients with urinary degradation ratios over 2 consistently had clinical evidence of mucopolysaccharide storage, whereas the patients with ratios below 2 had a minimal amount of such involvement.

Electrophoretic analysis of urinary concentrates as a supplement to the degradation ratio proved to be a simple and reliable method to determine the different mucopolysaccharides present in the urine. The high chondroitin sulfate content in the urine [11] necessi- tated the use of a buffer system (barium acetate) in which chondroitin sulfate could be distinguished as a separate band (Fig. 1). It was determined by electrophoresis that chondroitin sulfate was the major mucopolysaccharide in the urine from normal individuals. Minor components of normal urine (heparan sulfate, keratan sulfate, dermatan sulfate, and products of partial desulfation of chondroitin sulfate) described previously $[4,7]$ were not detected by this electrophoretic system. A minor Alcian blue-positive fraction $\left(A^{\prime}\right.$ in Fig. 1), which migrated more slowly than heparan sulfate, was characteristically seen but is not as yet chemically identified.

The major mucopolysaccharides in the urine from the Hurler patients were dermatan sulfate and heparan sulfate (Fig. 1). Thus, the electrophoretic pattern for urinary mucopolysaccharides proved to be a relatively easy way to determine whether there was any change in the distribution of different mucopolysaccharides in the urine.

In the analysis of urinary mucopolysaccharides, the determination of the degradation ratio coupled with the electrophoretic pattern of the CPC-precipitated mucopolysaccharides gave a basis to distinguish normal individuals from patients with clinical phenotypes diagnostic or suggestive of a genetic mucopolysaccharidosis $[8,9]$. As the degradation ratio reflects the total mucopolysaccharide metabolism [2], such a numerical value should be essential information in deciding whether any abnormality in mucopolysaccharide metabolism is involved in producing an abnormal clinical phenotype.

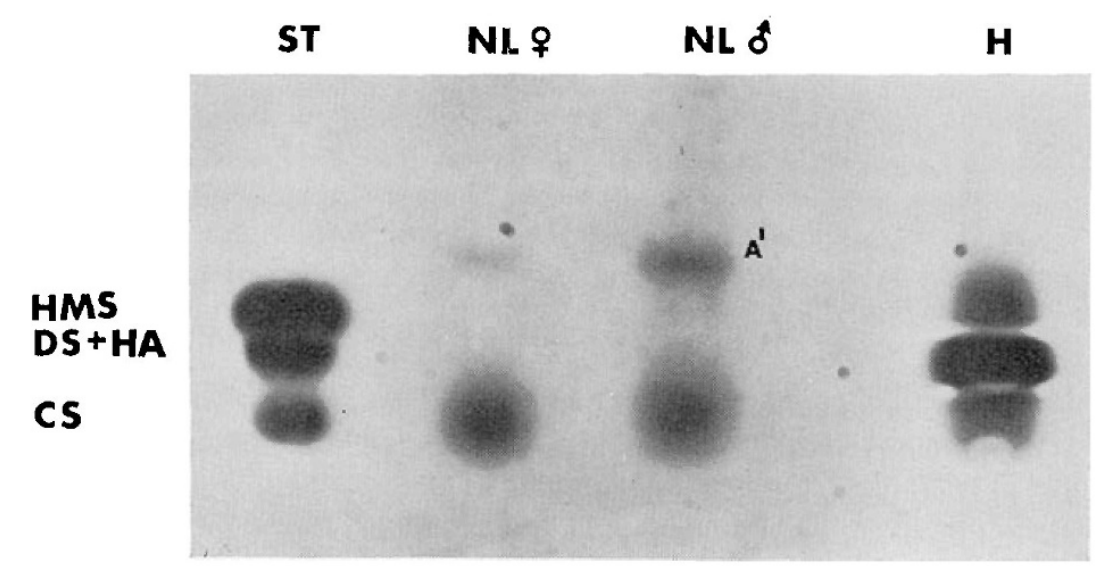

Fig. 1. Electrophoretic patterns of cetylpyridinium chloride-precipitated urinary mucopolysaccharides from two normal individuals ( $N L$ ) and a patient with the Hurler syndrome $(H)$ (run on cellulose acetate strip in barium acetate buffer). $S T$ : standards (provided by Dr. M. B. Mathews, Department of Pediatrics, University of Chicago); HA: hyaluronic acid; HMS: heparan sulfate; DS: dermatan sulfate; CS: chondroitin sulfate. A slow migrating band $\left(A^{\prime}\right)$ was observed in samples from normal individuals. 


\section{Summary}

The clegradation ratios for urinary mucopolysaccharides of 92 normal individuals (aged from birth to 25 years) were determined to establish the pattern of urinary mucopolysaccharides during development. The degradation ratio averaged $0.42 \pm 0.21$ and was not influenced by age, sex, or puberty. In contrast, the degradation ratios for urinary mucopolysaccharides for eight patients with a genetic mucopolysaccharidosis were above 1.0.

\section{References and Notes}

1. Bach, G., Friedman, R., Weissian, B., and Neufeld, E. F.: The defect in the Hurler and Scheie syndromes: Deficiency of $\alpha$-L-iduronidase. Proc. Nat. Acad. Sci. U. S. A., 69: 2048 (1972).

2. Di Ferrante, N., Neri, G., Neri, M. E., and Hogsett, W. E.: Measurement of urinary glycosaminoglycans with quaternary amonium salts: An extension of the method. Connect. Tissue Res., 1: 93 (1972).

3. Di Ferrante, N., Nichols, B. L., Donnely, P. V., Neri, G., Hrgovcic, R., ANd Berglund, R. K.: Induced degradation of glycosaminoglycans in Hurler's and Hunter's syndromes by plasma infusion. Proc. Nat. Acacl. Sci. U. S. A., 68: 303 (1971).

4. Di Ferrante, N., AND Rich, C.: The determination of acid mucopolysaccharides in urine. J. Lab. Clin. Med., 48: 491 (1956).

5. Drsche, A.: A new specific color reaction of hexuronic acids. J. Biol. Chem., 167: 189 (1947).
6. Dorfman, A., And Lorincz, A. E.: Occurrence of urinary acid mucopolysaccharides in the Hurler syndrome. Proc. Nat. Acad. Sci. U. S. A., 43: 443 (1957).

7. M.nley, G., Severn, M., and Hawksworth, J.: Excretion patterns of glycosaminoglycans and glycoproteins in normal human urine. J. Clin. Pathol., 21: 339 (1968).

8. McKusick, V. A: The nosology of the mucopolysaccharidoses. Amer. J. Med., 47: 730 (1969).

9. McKusick, V. A., Hussels, I. E., Howell, R. R., Neufeld, E. F., ANd Stevenson, R. E.: Allelism, non-allelism, and genetic compounds among the mucopolysaccharidoses. Lancet, $i$ : 993 (1972).

10. Tfeller, W. M., Burke, E. C., Rosfyear, J. W., and MCKEnZIE, B. F.: Urinary excretion of acid mucopolysaccharides in normal children and patients with gargoylism. J. Lab. Clin. Med., 59: 95 (1962).

11. WessLer, E.: Analytical and preparative separation of acidic glycosaminoglycans by elcctrophoresis in barium acetate. Anal. Biochem., 26: 439 (1968).

12. Model R-101, Beckman Instruments, Inc., Fullerton, Calif.

13. The authors wish to acknowledge the assistance of Beth $\mathrm{K}$. Rottrell and Sylvia D. Holley for her encouragement throughout the research.

14. The present address of C. Douglas is: Princeton University, Princeton, N. J.

15. This research was supported by a grant from The National Foundation-March of Dimes.

16. Requests for reprints should be addressed to: B. SHANNON Danes, M.D., Ph.D., The New York Hospital-Cornell Medical Center, 525 E. 68th St., New York, N. Y. 10021 (USA).

17. Accepted for publication April 17, 1973. 